\title{
El Estudio de las Preguntas Retóricas en el Cambio de Actitudes
}

\section{The Study of Rhetorical Questions in Attitude Change}

\author{
Ismael Gallardo, Camila Salazar, Camila Mendoza, Consuelo Rebolledo y Nadia Ramos \\ Universidad de Talca
}

\begin{abstract}
La investigación actual en psicología social ha mostrado que las preguntas retóricas (PRs) en contextos comunicativos afectan las actitudes que las personas tienen sobre un objeto, llevando a evaluaciones más positivas o negativas de él. La principal explicación de este efecto es que las PRs aumentan la elaboración cognitiva, lo que permite al receptor de la información distinguir la fuerza de los argumentos presentados en una propuesta. Como consecuencia, las personas pueden guiar sus pensamientos favorables o desfavorables en respuesta a ellas. El presente trabajo revisa la literatura sobre cambio de actitud y PRs e identifica en ella una serie de limitaciones procedimentales y de contenido. Se discute que controlando dichas limitaciones se pueden obtener nuevos resultados y, tal vez más importante, proponer nuevas explicaciones para ellos. Finalmente, se plantean nuevas áreas de investigación y potenciales aplicaciones de los resultados.
\end{abstract}

Palabras clave: actitudes, cambio, preguntas retóricas, revisión

\begin{abstract}
Current research in social psychology has shown that rhetorical questions (RQs) in communicative contexts affect the attitudes that people have about an object, leading either to positive or negative attitudes towards it. The main explanation for this effect is that RQs increase cognitive elaboration, which allows the receiver of the information to distinguish the strength of the arguments presented. As a consequence, people can guide their judgments based on the positive or negative thoughts generated as a response to RQs. This paper reviews the literature on attitude change and RQs and identifies procedural and content limitations not previously under control. It is discussed that controlling these limitations would make it possible to obtain new effects and potentially new explanations to them. In the final section new avenues for research and professional applications are proposed.
\end{abstract}

Keywords: attitudes, change, rhetorical questions, revision

Las preguntas retóricas (PRs) corresponden a aquellas que sugieren una cierta respuesta de manera implícita en su conformación lingüística (Howard, 1990; Petty, Cacioppo \& Heesacker, 1981; Zillmann, 1972) o, dicho de otro modo, corresponden a una afirmación con formato interrogativo (Domitrescu, 1992; Escandell, 1984; Gutiérrez, 1998; Igualada, 1994). Por ejemplo, la pregunta ¿A quién no le gustaría ganar la lotería? es retórica, dado que la afirmación implícita es "A todo el mundo le gustaría ganar la lotería". Dado que preguntar es una herramienta lingüística común en la interacción humana, el uso de este tipo de preguntas puede realizarse en todo contexto en el que sea necesario transmitir información a una persona o grupo. Su uso es bastante frecuente en diferentes campos profesionales, como la publicidad (Howard, 1991) o la terapia (Barraca, 2009; Ellis, 1962), lo que sugiere que es útil para cambiar opiniones y actitudes en las personas que las reciben. Sin embargo, la psicología social ha descubierto que la presencia de PRs puede tanto aumentar como reducir el cambio de actitudes, dependiendo de

Ismael Gallardo, Camila Salazar, Camila Mendoza, Consuelo Rebolledo y Nadia Ramos, Facultad de Psicología, Universidad de Talca, Chile.

El presente trabajo se enmarca en el proyecto FONDECYT 11070074 de responsabilidad del primer autor.

Agradecemos a Victoria González y Daniela Sánchez por su colaboración en la investigación desarrollada.

La correspondencia relativa a este artículo debe ser dirigida a Ismael Gallardo, Facultad de Psicología, Universidad de Talca, Avenida Lircay s/n, Talca, Chile. E-mail: igallardo@utalca.cl 
la fuerza de los argumentos presentados. Este doble efecto ha sido explicado fundamentalmente desde un enfoque de procesamiento de la información, según el cual el uso de PRs afecta a cuánto se piensa sobre un mensaje o propuesta (Petty, Cacioppo et al., 1981; Roskos-Ewoldsen, 2003). Pese a que estos resultados se replican consistentemente en la investigación contemporánea, existen limitaciones en dichos trabajos (procedimentales y estructurales), que no han sido reconocidas con anterioridad y que constituyen potenciales explicaciones para aquellos resultados. En este trabajo se plantea que el control de estas limitaciones puede llevar a importantes consecuencias teóricas, ya que anticiparía nuevos efectos y explicaciones. Estas nuevas predicciones podrían llevar a establecer estrategias de intervención más precisas en diferentes ámbitos profesionales.

En los párrafos siguientes se describirá el contexto teórico en el que se enmarca el estudio de las PRs en psicología social y los principales resultados encontrados en la investigación. Luego se discutirá sobre las limitaciones de estos resultados, así como potenciales explicaciones alternativas y nuevos efectos. Finalmente, se analizará el impacto tanto teórico como práctico de estas predicciones.

\section{Cambio de Actitudes}

Las actitudes se definen como evaluaciones positivas o negativas que las personas realizan ante diferentes objetos sociales y que reciben el nombre genérico de objetos de actitud (Briñol, De la Corte \& Becerra, 2001; Cárdenas \& Barrientos, 2008). El cambio de actitudes ocurre cuando una persona o grupo modifica estas evaluaciones después de recibir una información de otra persona o grupo en un contexto determinado (Petty \& Briñol, 2010; Petty \& Wegener, 1998). El desarrollo teórico y empírico de este campo de estudio comenzó con el trabajo pionero de los investigadores de la Escuela de Yale (Hovland, Janis \& Kelley, 1953), el cual buscaba conocer los efectos de las variables comunicativas sobre las evaluaciones que se realizan ante diferentes objetos de actitud (e.g., ¿es más probable el cambio de actitudes cuando las personas están de buen humor?). Estos primeros estudios se basaban en un modelo en el que el cambio de actitudes dependía de una serie de etapas de aprendizaje del mensaje entregado (Hovland et al., 1953).

Posteriormente, las investigaciones en el cambio de actitudes mostraron que el aprendizaje de un mensaje no siempre lleva a una modificación de las actitudes y, además, que estas pueden cambiar aun cuando una persona no aprenda el contenido del mensaje (Chaiken, 1980; Petty, Ostrom \& Brock, 1981). Así se dio paso a un enfoque del cambio de actitudes basado en la idea de que las personas son sujetos activos en el proceso comunicativo y, por tanto, interpretan y responden a la propuesta recibida, independientemente de la intención que esta posee (Greenwald, 1968). Entonces, el cambio de actitudes dependerá de los pensamientos (respuestas cognitivas) que los individuos generen cuando reciben una información. En la actualidad se entiende que dos dimensiones de los pensamientos son fundamentales para que exista un cambio de actitudes (Chaiken, Liberman \& Eagly, 1989; Petty \& Cacioppo, 1986): la dirección y la cantidad de pensamientos generados.

La dirección de los pensamientos alude a lo favorable o desfavorable que estos resultan como respuesta a un mensaje, siendo ampliamente demostrado que mientras más favorables son estos mayor es su efecto persuasivo (Greenwald, 1968; Petty, Ostrom et al., 1981; Petty \& Wegener, 1998). La cantidad de pensamiento hace referencia al número de pensamientos que las personas generan al elaborar la información persuasiva que reciben. Mientras más se piensa (elabore) un mensaje, los cambios actitudinales se producen a través de la llamada ruta central, caracterizada por condiciones en las que las personas poseen motivación y capacidad para elaborar la información presentada. En cambio, cuando las personas tienden a pensar poco 
o nada sobre un mensaje, entonces los cambios actitudinales se generan a través de la llamada ruta periférica, caracterizada por condiciones en las que las personas no poseen ni motivación ni capacidad para elaborar la información presentada. A diferencia de las perspectivas lingüísticas que ponen énfasis en las intenciones del emisor de un mensaje (González, 2011; White, 2003), este enfoque plantea que la clave para comprender el cambio de actitudes implica atender al receptor de la información. Los modelos explicativos principales en los que se basa la gran mayoría de los trabajos en cambio de actitudes son el modelo de probabilidad de elaboración (Elaboration Likelihood Model, ELM; Petty \& Cacioppo, 1986) y el modelo heurístico sistemático (HSM; Chaiken et al., 1989). Aun cuando los supuestos de ambos modelos son similares, en este trabajo se utiliza el ELM, debido a que el estudio de las PRs se ha desarrollado principalmente bajo esta perspectiva (Petty \& Briñol, 2010).

Un importante aporte del ELM para la comprensión del cambio de actitudes es proponer que las variables clásicas del proceso comunicativo (emisor, receptor, mensaje, canal, contexto; Briñol et al., 2001) no afectan de manera única al cambio de actitudes, sino que lo hacen siguiendo múltiples roles (Petty \& Wegener, 1998); esto es, que una misma variable (e.g., la credibilidad de la fuente de un mensaje) puede llevar al cambio de actitudes a través de diferentes procesos psicológicos, dependiendo de la cantidad de pensamiento que las personas dediquen a la elaboración del mensaje (Petty \& Briñol, 2010). Hasta la fecha se pueden identificar diferentes roles que adoptan las variables para afectar el cambio de actitudes (Briñol \& Petty, 2009; Petty \& Wegener, 1998). Por ejemplo, cuando la probabilidad de elaboración es baja (i.e., cercana a la ruta periférica), las variables del proceso comunicativo pueden actuar sirviendo como una clave sencilla de decisión (e.g., si un experto avala esta decisión, probablemente sea buena). Por otra parte, cuando la probabilidad de elaboración no está limitada a ser alta o baja, una variable puede influir en la cantidad de elaboración dedicada, aumentándola o reduciéndola (e.g., si un experto concuerda con esto, quizás debiera prestar atención a lo que dice). Por último, cuando la probabilidad de elaboración es alta (i.e., cercana a la ruta central), una variable puede servir como un argumento más de la propuesta (e.g., si un experto avala esta propuesta, es otra buena razón para avalarla también); puede sesgar el procesamiento de la información (e.g., si un experto avala esta propuesta, probablemente sea buena y todo lo que ella plantee sea bueno) o puede afectar a la validez de los propios pensamientos (e.g., si la fuente no es experta, puede que lo que haya pensado no sea tan válido como creía).

Este modelo de cambio actitudinal permite, entonces, controlar y predecir resultados específicos para las diferentes variables persuasivas como, por ejemplo, aquellas relativas al emisor (e.g., el atractivo; DeBono \& Harnish, 1988), al receptor (e.g., el estado de ánimo; Briñol, Petty \& Barden, 2007), al canal (e.g., escrito; Burnkrant \& Howard, 1984) o al mensaje (e.g., que contiene o no preguntas retóricas; Petty, Cacioppo et al., 1981).

\section{El Estudio de las PRs en el Cambio de Actitudes}

El estudio de las PRs en el cambio de actitudes se ha realizado siguiendo una metodología experimental. En términos generales, los trabajos controlan la presencia o ausencia de este tipo de preguntas en un contexto persuasivo (por ejemplo, presentando información sobre un objeto en particular cuyo contenido incluye $-\mathrm{O}$ no- PRs), siendo la variable dependiente las actitudes que las personas poseen ante la información presentada. El primer estudio en el que este formato fue sometido a prueba fue realizado por Zillmann en 1972, creando una situación experimental en donde un grupo de estudiantes de psicología recibió el caso de un joven acusado de asesinato, a quien se debía asignar una sentencia de prisión. Los participantes recibieron información inicial que les llevaba a tener actitudes favorables, desfavorables o neutras hacia el joven. Posteriormente, debían escuchar los argumentos de la defensa. Para la mitad de los participantes, estos argumentos eran presentados resumidos en una pregunta (e.g., Johnny era 
un niño pacífico, ¿no es así?), mientras que la mitad restante no tenía este formato interrogativo $^{1}$. Los resultados mostraron que las personas tendieron a otorgar menos años de prisión en la condición de pregunta que en la de control, independientemente de la dirección de las actitudes iniciales. Zillmann argumentó que este efecto podría deberse a una serie de circunstancias de aprendizaje que enseñaban a las personas que los buenos argumentos eran acompañados por preguntas de este tipo, razón por la cual su mera inserción era indicador de una buena propuesta (Zillmann, 1972; Zillmann \& Cantor, 1974). Por tanto, la presencia de PRs llevaba a actitudes favorables hacia el objeto.

En una investigación posterior, Petty, Cacioppo et al. (1981) mostraron que las PRs pueden llevar a actitudes favorables o desfavorables, debido a que estas preguntas pueden aumentar la cantidad de pensamientos generados y relacionados con la propuesta, especialmente si las personas no tienden a pensar demasiado sobre la información que reciben (i.e., bajas en necesidad de cognición [NC]; Cacioppo, Petty, Kao \& Rodriguez, 1986). De este modo, un mensaje fuertemente argumentado lleva a actitudes más favorables, mientras que uno débilmente argumentado lleva a actitudes más desfavorables. Sin embargo, cuando las personas tienden naturalmente a pensar de manera sistemática sobre la información social (i.e., altos en NC), este aumento de la cantidad de pensamientos generados por la presencia de PRs puede llevar a que las personas distraigan su atención del objeto de actitud. De este modo, no son capaces de distinguir la fuerza de los argumentos presentados, no existiendo diferencias en las actitudes. En el estudio de Petty, Cacioppo et al. (1981) un grupo de estudiantes de psicología recibió un mensaje auditivo fuertemente argumentado a favor de instalar una política universitaria, mientras que otro grupo recibió el mismo mensaje, pero débilmente argumentado. Simultáneamente, para la mitad de los participantes estos argumentos se resumían en una serie de PRs, mientras que para el resto, en aseveraciones (statements). Los resultados mostraron que las PRs llevaron a actitudes más favorables hacia la propuesta universitaria cuando los argumentos presentados eran muy convincentes y a más desfavorables cuando los argumentos presentados eran poco convincentes. Este efecto de la calidad de los argumentos se encontró especialmente en aquellas personas bajas en NC. En personas altas en NC la presencia de PRs redujo el efecto de la calidad de los argumentos del mensaje, de modo tal que las actitudes en respuesta a la propuesta fuerte o débil de la política universitaria no se distinguieron significativamente.

El experimento de Petty, Cacioppo et al. (1981) mostró que las PRs pueden, dependiendo de ciertas condiciones, aumentar o reducir la persuasión. Estos efectos han sido explicados en función del impacto que tienen las PRs sobre la elaboración cognitiva de la propuesta (Areni, 2003; Petty \& Wegener, 1998). Para el caso de las personas bajas en NC, la PR incrementó el efecto de la calidad de los argumentos del mensaje (comparado con un grupo control), lo que es una evidencia del aumento en la elaboración del mismo. Sin embargo, para las personas altas en NC, la PR redujo el efecto de la calidad de los argumentos, indicando que las personas no han elaborado la información recibida. Esto generó un importante avance en la comprensión de los procesos psicológicos a la base del efecto de las PRs, ya que mostró que no solo genera actitudes positivas (como lo sugería Zillmann, 1972), sino que, bajo ciertas circunstancias, actitudes negativas. En la investigación en psicología social en los últimos 30 años, esta explicación ha sido consistentemente validada en función de los diferentes efectos encontrados, siendo posible encontrar moderadores del efecto principal, pero no así nuevos mediadores. Dicho de otro modo, aun cuando las PRs afectan en mayor o menor medida la elaboración del mensaje dependiendo de ciertas condiciones, la explicación de base sigue siendo la misma.

Así, por ejemplo, Burnkrant y Howard (1984) mostraron que cuando los mensajes son escritos (una variable del canal comunicativo), las PRs aumentan la elaboración cognitiva del

\footnotetext{
Si bien la pregunta usada es aseverativa, Zillmann (1972) argumentó que este tipo de preguntas utilizadas en personas que no podían responder a la interrogación del emisor funcionaba de manera retórica, en la medida en que resumía una serie de afirmaciones previas que llevaban implícitamente la respuesta a la interrogación realizada. Zillmann (1972) plantea que este efecto debería mostrarse también en preguntas formuladas de manera retórica.
} 
mensaje y, con ello, el efecto de la calidad de los argumentos del mismo, independientemente de cuánto esté pensando el receptor. Munch y Swasy (1988) mostraron que cuando el objeto de actitud es novedoso, la presencia de una PR reduce la elaboración del mensaje. Ahluwalia y Burnkrant (2004) encontraron que la evidencia (versus sutileza) de la presentación de una PR en una propuesta comunicativa afecta el foco de atención del receptor. Según ello, la atención en los argumentos del mensaje se daría cuando las PRs no son evidentes para quien recibe la información. En cambio, cuando la presencia de PRs es evidente (controlado a partir del uso exagerado de las mismas), la atención del receptor se desviaba desde los argumentos del mensaje hacia la fuente de persuasión (Swasy \& Munch, 1985). Además de estos resultados, se ha encontrado que las PRs poseen importantes consecuencias en las actitudes formadas a partir de su inclusión en un contexto persuasivo. Por ejemplo, Blankenship y Craig (2006) encontraron que las personas no solo elaboran más profundamente la información recibida en presencia de PRs, sino que también contra-argumentan más que en una condición de control en presencia de un mensaje que se opone a las actitudes previamente formadas. Como se puede observar, los efectos de las PRs pueden ser diferentes dependiendo de ciertas condiciones, pero todas aluden a un mismo proceso subyacente: la elaboración cognitiva.

\section{Algunas Limitaciones en el Estudio de las PRs en Psicología Social}

Tanto las investigaciones clásicas como las más recientes sobre PRs y cambio de actitud proveen resultados aparentemente confiables a través del tiempo. Pese a ello, poseen algunos problemas que hasta hoy no han sido evaluados. En este trabajo se plantea que el control de dichos problemas podría llevar a explicar los resultados hasta ahora encontrados, descubrir nuevos efectos y encontrar nuevas explicaciones. Para ordenar esta información, las diferentes limitaciones se han agrupado en aquellas referentes al procedimiento de investigación y aquellas referentes al contenido de las preguntas.

Respecto del procedimiento, existen discrepancias en los estudios en la forma de controlar experimentalmente la presencia de PRs en un contexto persuasivo. En algunos trabajos son presentadas como resúmenes de argumentos previamente planteados (e.g., Petty, Cacioppo et al., 1981), como viñetas (e.g., Swasy \& Munch, 1985) o como encabezados y resúmenes finales ocupados en conjunto (e.g., Ahluwalia \& Burnkrant, 2004). Homologar las formas de manipular experimentalmente las PRs es imprescindible para comparar los estudios, puesto que los diferentes resultados pueden deberse, simplemente, al formato de presentación. Así, por ejemplo, las investigaciones no han considerado si la presencia de PRs en forma de viñetas afecta de manera separada o aditiva al cambio de actitudes. Cuando se presenta una serie de estas, no es posible saber si el efecto sobre las actitudes se debe a la primera viñeta, a la suma de las dos primeras, a la lectura o recuerdo solo de la última que se presentó o a un efecto de todas ellas en conjunto. De acuerdo con lo anterior, es posible que las PRs lleven al cambio de actitudes debido a un efecto de primacía, a un efecto de recencia, a la adición de los estímulos o a otra alternativa no explorada (Higgins, 2000). Por tanto, para establecer con claridad la consistencia de los datos mostrados en las investigaciones se hace necesario homologar la forma de presentación de las PRs.

También el momento en que las preguntas se presentan dentro del contexto comunicativo (antes o después de la recepción de una información) es variado en los trabajos hasta ahora realizados, y su efecto no ha sido dilucidado con claridad. La mayoría de estos trabajos (excepto Burnkrant \& Howard, 1984) presenta, en sus diversas formas, las PRs durante o después de la emisión de los argumentos. Esta sencilla variación procedimental es muy relevante, debido a que estudios recientes en el cambio de actitudes han demostrado que el momento en que se presentan las variables del proceso comunicativo afecta la evaluación que se realiza de un objeto en particular, a través de procesos psicológicos que son diferentes en un caso y otro (Petty \& Briñol, 2010). A modo explicativo, si una variable es presentada antes de una información persuasiva, dicha variable afectará las respuestas cognitivas (pensamientos) que las personas 
generen hacia el mensaje. Así, un pensamiento del tipo "me gusta el fútbol" o "ese producto es bueno" puede estar afectado por la presencia previa de una variable persuasiva (e.g., el estado de ánimo positivo; Briñol, Petty \& Barden, 2007). Sin embargo, cuando las personas ya han generado pensamientos ante un mensaje (favorables o desfavorables), la presencia de una variable persuasiva no afectará su elaboración, sino que influirá en aquellos pensamientos que se tengan sobre los pensamientos ya generados o en aquellos pensamientos acerca de los procesos de generación de pensamiento, como, por ejemplo, “irealmente me gusta el fútbol?” o "confío en mis pensamientos acerca de la bondad de este producto" (Jost, Kruglanski \& Nelson, 1998). En este caso, una variable persuasiva ya no afectará las actitudes a través de un proceso relacionado a la generación de pensamientos, sino a uno vinculado a la metacognición, como, por ejemplo, la validez que se le otorgan a los propios pensamientos (Petty, Briñol \& Tormala, 2002). Ejemplos de variables que pueden actuar a través de uno u otro proceso, dependiendo del momento de presentación, son el rol que posee el poder del receptor (de entregar recompensas y castigos; Briñol, Petty, Valle, Rucker \& Becerra, 2007), el rol de la afirmación de la identidad del receptor (Briñol, Petty, Gallardo \& DeMarree, 2007), el rol de la credibilidad que posee la fuente de información (Tormala, Briñol \& Petty, 2006), el rol de la mayoría o minoría numérica (Horcajo, Petty \& Briñol, 2010), entre otros. En el contexto del estudio de PRs, solo Howard (1990) ha controlado experimentalmente el momento en que se presentaba una PR, mostrando que estas afectaban a las actitudes, sobre todo cuando se entregaban después de un mensaje. Sin embargo, su explicación para estos efectos también está vinculada a la elaboración cognitiva, lo que impide dar cuenta de resultados previos que son contrarios a los que encontró (e.g., Petty, Cacioppo et al., 1981). En este sentido, se hace necesario controlar adecuadamente el momento en que se presenta una PR en un contexto persuasivo, para identificar con claridad los procesos responsables.

Además de estas limitaciones procedimentales, existen otras relacionadas con el contenido de las PRs. Para dar cuenta de estas con mayor claridad, se usará como referencia la pregunta “¿a quién no le gustaría ganar la lotería?”, en la que se afirma implícitamente que "todo el mundo querría ganar la lotería". La investigación previa ha centrado su análisis en el efecto de este tipo de formato lingüístico como un todo, sin considerar los efectos que algunas de sus partes estructurales podrían llegar a tener por sí mismas. Por ejemplo, la PR anterior implica que la afirmación que se encuentra implícita en ella alude a una mayoría numérica que adhiere a la propuesta ("todo el mundo..."). Si el formato retórico fuera simplemente el responsable de los efectos encontrados, no debería ser relevante que una pregunta afirme implícitamente a una minoría en ella como “¿a quién le gustaría ganar la lotería?” o “¿realmente alguien querría ganar la lotería?" (que aluden a que "nadie querría ganar la lotería"). Sin embargo, la investigación actual ha mostrado que la referencia a una mayoría o minoría numérica en un contexto comunicativo no solo lleva a diferentes efectos sobre las actitudes resultantes, sino también que estos se pueden deber a procesos psicológicos diferentes. Horcajo et al. (2010, experimento 3) encontraron recientemente que hacer accesible el contenido de un objeto de actitud que es apoyado por una mayoría o minoría numérica podía tener efectos opuestos en la evaluación que una persona pueda dar a ese objeto en particular. Así, por ejemplo, cuando se generan pensamientos favorables y luego se anuncia que dichas ideas son compartidas por muchas personas, las actitudes son más favorables que cuando se anuncia que son compartidas por pocas. Sin embargo, este efecto se invierte cuando las personas reciben esta información antes de un mensaje. En este caso, en la condición de mayoría se observan actitudes y pensamientos más desfavorables que en la condición de minoría. El análisis conjunto de este y otros estudios realizados por los autores mencionados mostró que estos efectos paradójicos se deben a que están causados por procesos psicológicos diferentes: para el primer caso, un proceso metacognitivo conocido como autovalidación (Briñol \& Petty, 2009) y para el segundo, una reducción de la elaboración de los pensamientos generados como respuesta a la información presentada. Siguiendo estos resultados, el efecto de la PR de referencia puede deberse a su significado (la alusión a una mayoría o minoría) y no a su formato. 
Otra posible explicación de los efectos encontrados en la investigación y que implica un aspecto estructural de las PRs es la presencia de una negación (“¿a quién no le gustaría...?). Todos los estudios realizados en cambio de actitudes (excepto Howard, 1990) incorporan en sus estímulos experimentales PRs que contienen una negación (Ahluwalia \& Burnkrant, 2004; Areni, 2003; Blankenship \& Craig, 2006; Munch \& Swasy, 1988; Petty, Cacioppo et al., 1981; Swasy \& Munch, 1985; Zillmann, 1972), lo que no se ha considerado hasta el momento como potencial causal de sus consecuencias sobre el cambio actitudinal. Diferentes trabajos han mostrado que oraciones con negación ("Sebastián no desea salir") hacen saliente el esquema o unidad cognitiva ("Sebastián desea salir"), para luego ser usada como etiqueta, siempre y cuando exista la motivación suficiente para ello. De esta forma, al integrarse a la oración, la suposición central se considera como inapropiada (Just \& Carpenter, 1976; Mayo, Schul \& Burnstein, 2004). En un estudio, Clark y Chase (1972) solicitaron a sus participantes que verificaran el tipo de oración que describía con más precisión una imagen, presentándoles para ello una serie de oraciones que contenían o no una negación en su estructura. Cuando los individuos escogían una oración con una negación, tomaban mucho más tiempo en lograr la descripción que cuando escogían una oración que no la contenía, indicando que la información a procesar era mayor en esa condición. En una línea similar a la anterior, los estudios en los que a las personas se les pide que nieguen un tipo de pensamiento (e.g., "no pensar en osos blancos"; Wegner, 1989), paradójicamente piensan más en él, dado que para negarlo es necesario, en primer lugar, activar la etiqueta de contenidos, para luego descartarla (Wegner, 1989; véase también Lea \& Mulligan, 2002). Como ya se ha revisado, el aumento en la elaboración cognitiva es la principal consecuencia de la presencia de una PR en un contexto comunicativo, al igual que para el caso de la negación. Por tanto, ¿a qué se debe el efecto de una PR: a su formato o a la presencia de una negación en ella?

Recientemente, Gallardo, Briñol y Salazar (2012) evaluaron esta idea, controlando experimentalmente la presencia o ausencia de una negación en una PR dentro de un contexto persuasivo. Solicitaron a la mitad de los participantes generar pensamientos favorables hacia la construcción de viviendas espaciales, y al resto, desfavorables. Posteriormente, la mitad de los participantes recibieron una PR que contenía una negación (“¿a quién no podría gustarle la propuesta de construcción de viviendas en Marte?"), mientras que la mitad restante recibió una PR sin negación (“¿a quién podría gustarle la propuesta de construcción de viviendas en Marte?”). Los resultados mostraron que, a pesar de que las personas generaron pensamientos favorables o desfavorables hacia el objeto de actitud, solo aquellas que recibieron una pregunta sin negación utilizaron dichos pensamientos para evaluar el objeto. En cambio, cuando las PRs contenían una negación, se redujo el efecto de los pensamientos previamente generados, no siendo utilizados para realizar la evaluación del objeto. Este patrón de resultados sugiere fuertemente que la PR con negación redujo la validez que las personas otorgaban a sus pensamientos previos.

El trabajo descrito tiene una serie de ventajas en comparación con otros anteriores. En primer lugar, controla las dos críticas centrales descritas previamente sobre el procedimiento utilizado en trabajos anteriores. Es decir, mantiene constante el formato de presentación de las PRs y solo modifica la presencia o ausencia de una negación. Además, controla el momento en que se presenta una PR (después de la generación de pensamientos). Al manejar estas variables, los autores pudieron discutir que el efecto encontrado puede deberse a un proceso psicológico diferente a la elaboración cognitiva. Dado que las PRs han sido manipuladas después de la generación de pensamientos, es difícil que su efecto se deba a un proceso relacionado con la elaboración de los mismos. Más probable, sin embargo, es que derive de un proceso metacognitivo (o cognitivo de segundo orden; Jost et al., 1998) concretamente, el de autovalidación (Petty \& Briñol, 2010). La autovalidación plantea que la dirección de los pensamientos afectará las actitudes siempre y cuando las personas les otorguen validez (confíen). En cambio, si las personas no otorgan validez a sus pensamientos, entonces estos no serán utilizados para realizar el juicio posterior (Bohner \& Dickel, 2011; Briñol \& Petty, 2009). En línea con esta idea, las 
PRs con negación parecen reducir la validez que se le otorga a los pensamientos que se poseen en torno a un objeto de actitud. Este tipo de consecuencias metacognitivas de las PRs no se ha encontrado en la investigación hasta ahora realizada, constituyendo un potencial nuevo proceso psicológico por el cual esta variable puede afectar la persuasión (para una revisión, véase Bohner \& Dickel, 2011; Briñol \& Petty, 2009).

Es posible que estos resultados no se deban a la presencia de la negación, sino a que la PR posee la característica fundamental de negar implícitamente lo que afirma (Igualada, 1994). Dado que el formato retórico se mantiene constante entre las condiciones, esta explicación parece menos plausible (ambas PRs niegan). Por otra parte, si es el contenido negado lo que genera los efectos, entonces no debería tener diferencias con una condición de control que sea semánticamente similar. En otro estudio, Salazar (2010) comparó la PR con negación con su homólogo semántico ("a todo el mundo le gusta la propuesta..."), mostrando los mismos efectos antes descritos. Es decir, aun cuando ambas condiciones tenían el mismo significado, solo ante una negación las personas no utilizaron los pensamientos previamente generados. Los resultados mencionados sugieren fuertemente que el simple uso de una negación puede dar cuenta de los efectos de las PRs en persuasión y, además, que el aumento de la elaboración cognitiva puede no ser la única explicación disponible para ellos.

\section{Futuras Investigaciones}

El estudio futuro de este tipo de preguntas se puede desarrollar tanto en el área teórica como práctica. Una primera línea de trabajo teórico implica avanzar en el conocimiento de los efectos que poseen otros elementos de las PRs que se encuentran en la investigación y siguen sin ser estudiados. En los párrafos anteriores se ha mostrado que el efecto que tiene una PR sobre las actitudes de las personas puede deberse a múltiples razones y que, en último término, el formato retórico puede ser una variable más entre todas aquellas que se han planteado. Por tanto, cabe preguntarse si realizar PRs es algo diferente de usar otro tipo de preguntas como, por ejemplo, informativas (Gutiérrez, 1998). En ellas no se afirma algo de manera implícita, sino que se realiza un requerimiento de respuesta al receptor (e.g., "¿te gustaría ganar la lotería?"). Este tipo de preguntas podría llevar a un mayor procesamiento que una PR, debido a la activación de las etiquetas de juicio asociadas con lo que se pregunta o desea conocer (aumentando su accesibilidad; Higgins, 2000). También es posible que la intención que el receptor percibe del emisor de la pregunta (por ejemplo, una orden, un sarcasmo o una ironía; Cepeda \& Rosas, 2007; Gibbs, 2000) genere otro tipo de efectos, como actitudes favorables (para complacer la orden; Cialdini \& Trost, 1998) o desfavorables (por reactancia a la ironía; Brehm, 1966). En un polo más extremo aún, cabe la posibilidad de que la sola presencia de signos de interrogación afecte la forma de procesar o validar la información que se recibe, dado que es un indicador de un requerimiento por parte del emisor o una solicitud para pensar sobre lo que ya se ha pensado. Próximos trabajos deberán evaluar estas u otras variable no identificadas.

Una segunda área teórica se refiere a nuevas explicaciones por descubrir para el efecto de las PRs en persuasión. En el marco del ELM, una PR podría llevar a efectos sobre las actitudes, siguiendo otros procesos psicológicos, dependiendo de las condiciones de motivación y capacidad del receptor. Por ejemplo, en condiciones de baja motivación y capacidad (e.g., baja relevancia personal), la presentación de una PR antes de la recepción de una información puede llevar a un aumento de la elaboración cognitiva y, por tanto, a un mayor efecto de la calidad de los argumentos presentados en un mensaje (Petty, Cacioppo et al., 1981). Sin embargo, también es posible que una PR lleve a persuasión a través de mecanismos de ruta periférica como, por ejemplo, sirviendo como heurístico o clave sencilla de decisión (Chaiken, 1980; Schwarz et al., 1991). En este caso, no será la fuerza de los argumentos la responsable de los efectos sobre las actitudes, sino la mera inferencia de que el mensaje es bueno porque este tipo de preguntas lo utilizan personas expertas (Zillmann, 1972). Por otro lado, si la motivación y capacidad no está limitada a ser alta o baja, entonces una PR podría servir como una variable que aumente la 
probabilidad de elaborar profundamente la información, tal como se ha observado en investigaciones previas (Burnkrant \& Howard, 1984). En condiciones de alta motivación y capacidad, una PR puede servir como un argumento más para la propuesta entregada (Kruglanski et al., 2005), llevar a corrección de los juicios previamente realizados (Wegener \& Petty, 1995) o, si es entregada después de la generación de pensamientos, afectar la validez que las personas le otorgan a estos (Briñol \& Petty, 2009). Hasta ahora se han estudiado explicaciones relativas al efecto de las PRs sobre la elaboración del pensamiento o sobre la validez que se le otorga a los mismos. No existen, hasta el conocimiento de los autores de este artículo, efectos de las PRs explicados a través de procesos relacionados con la ruta periférica, lo que podría ser objeto de atención de próximos estudios.

El efecto de las PRs no se limita a situaciones experimentales o teóricas, sino que sienta las bases para desarrollar estrategias de intervención social. De este modo, los principios fundamentales que subyacen a los efectos antes descritos se pueden aplicar en cualquier situación que involucre la transmisión de información. Por ejemplo, en publicidad, la instalación de un nuevo producto puede verse beneficiada por la inclusión de una PR al comienzo del anuncio del mismo. Dado que este procedimiento puede generar un aumento en la elaboración de la información que se presentará a continuación, se hace necesario también generar un mensaje muy convincente. Así, al usar PRs parece menos conveniente la incorporación de otros elementos, como la generación de estados de ánimo positivos (a través de chistes) o el uso de fuentes creíbles (e.g., expertos), dado que pueden reducir la elaboración de la información a entregar (Tormala et al., 2006). Controlar la fuerza de los argumentos de un mensaje en este contexto es fundamental, dado que un mensaje considerado débil puede generar pensamientos desfavorables y, si las personas están pensando (debido a la inclusión de una PR), las actitudes posteriores serán probablemente negativas. Por otro lado, la mantención de un producto en el mercado puede ser una oportunidad importante para validar los pensamientos que se tiene en torno al mismo. Si las personas recuerdan los atributos positivos del producto, una PR podría validarlos (e.g., “¿acaso alguna vez se ha construido algo mejor?”). Cabe recordar que el uso de negaciones en este contexto podría mermar el impacto de los pensamientos previos, por lo que es necesario tener especial cuidado en el diseño de estos dispositivos.

Otro contexto muy importante en que se puede aplicar la lógica multiproceso de las PRs es en la terapia, específicamente, como estrategia para mejorar las habilidades terapéuticas. Por ejemplo, en etapas iniciales del proceso terapéutico, un terapeuta podría encontrarse con un paciente reticente a contar los motivos de su consulta. En este caso, convendrá que el terapeuta le ayude a centrar sus pensamientos en estos motivos, ayudado por el aumento en el procesamiento de información que generaría una PR (e.g., "¿quién no ha pensado alguna vez en cambiar algo de sí mismo?"). En cambio, la inclusión de una PR durante el proceso terapéutico podría ayudar a validar o invalidar pensamientos previos adecuados o inadecuados en relación al objeto de consulta. Así, una PR que incluya una negación podría servir para reducir la validez que poseen pensamientos positivos hacia la realización de conductas inadecuadas (e.g., consumo de drogas), como, por ejemplo, “¿quién no querría comenzar a consumir drogas?”. El efecto se genera aun cuando la afirmación implícita es "todo el mundo querría comenzar a consumir drogas", debido a que la activación de los pensamientos en relación con el objeto más la etiqueta de negación deberían reducir la utilidad que las personas perciben en dichos pensamientos. Sin embargo, una PR sin esta etiqueta (e.g., “quién querría comenzar a consumir drogas?”) podría generar mayor validez en los pensamientos previos. De este modo, realizar preguntas en contextos terapéuticos tendrá efectos positivos o negativos, dependiendo del tipo de pregunta realizada, un asunto que no es considerado en la enseñanza de estas herramientas.

\section{Comentarios Finales}

La presente revisión muestra que en el estado actual de la investigación en PRs no existe controversia alguna sobre las potenciales causas que tiene el efecto de esta variable sobre el 
cambio de actitudes. Hasta donde los autores de este trabajo tienen conocimiento, este es el primero en el cual se discuten explicaciones alternativas a la elaboración cognitiva, incorporando, así, conocimiento novedoso para la investigación básica y para la aplicación profesional. Adicionalmente, es importante mencionar que no existen trabajos en psicología social que hayan estudiado el efecto de las PRs sobre el cambio de actitud en idioma español. No obstante, de acuerdo a la literatura (Gutiérrez, 1998), esta discrepancia idiomática no debería alterar las propiedades lingüísticas de una PR, razón por la cual las predicciones aquí realizadas deberían ser comparables con aquellos trabajos realizados en idioma inglés. Estos planteamientos sirven para abrir una discusión sobre los efectos psicológicos que tiene la inclusión de estos elementos lingüísticos en un contexto comunicativo, los que, como se ha visto, pueden no tener relación alguna con lo que el emisor quiere informar, sino más bien con la manera en que el receptor analiza la información o piensa sobre lo ya analizado. Finalmente, las implicaciones para distintos ámbitos profesionales no se limitan a la publicidad o la terapia, sino a todo contexto en el que se desee entregar información.

\section{Referencias}

Ahluwalia, R. \& Burnkrant, R. E. (2004). Answering questions about questions: A persuasion knowledge perspective for understanding the effects of rhetorical questions. Journal of Consumer Research, 31, 26-42. doi:10.1086/383421

Areni, C. S. (2003). The effects of structural and grammatical variables on persuasion: An elaboration likelihood model perspective. Psychology and Marketing, 20, 349-375. doi:10.1002/mar.10077

Barraca, J. (2009). Habilidades clínicas en la terapia conductual de tercera generación. Clínica y Salud, 20, 109 -117.

Blankenship, K. L. \& Craig, T. Y. (2006). Rhetorical question use and resistance to persuasion: An attitude strength analysis. Journal of Language and Social Psychology, 25, 111-128. doi:10.1177/0261927X06286380

Bohner, G. \& Dickel, N. (2011). Attitudes and attitude change. Annual Review of Psychology, 62, 391-417. doi:10.1146/ annurev.psych.121208.131609

Brehm, J. W. (1966). A theory of psychological reactance. San Diego, CA: Academic Press.

Briñol, P., De La Corte, L. \& Becerra, A. (2001). Qué es persuasión. Madrid, España: Biblioteca Nueva.

Briñol, P. \& Petty, R. (2009). Persuasion: Insights from the self-validation hypothesis. En M. Zanna (Ed.), Advances in experimental social psychology (Vol. 41, pp. 69-118). New York, NY: Academic Press. doi:10.1016/S00652601(08)00402-4

Briñol, P., Petty, R. E. \& Barden, J. (2007). Happiness versus sadness as a determinant of thought confidence in persuasion: A self-validation analysis. Journal of Personality and Social Psychology, 93, 711-727. doi:10.1037/00223514.93.5.711

Briñol, P., Petty, R. E., Gallardo, I. \& DeMarree, K. G. (2007). The effect of self-affirmation in nonthreatening persuasion domains: Timing affects the process. Personality and Social Psychology Bulletin, 33, 1533-1546. doi:1 $0.1177 / 0146167207306282$

Briñol, P., Petty, R. E., Valle, C., Rucker, D. D. \& Becerra, A. (2007). The effects of message recipients' power before and after persuasion: A self-validation analysis. Journal of Personality and Social Psychology, 93, 1040-1053. doi:10.1037/0022-3514.93.6.1040

Burnkrant, R. E. \& Howard, D. J. (1984). Effects of the use of introductory rhetorical questions versus statements on information processing. Journal of Personality and Social Psychology, 47, 1218-1230. doi:10.1037/00223514.47.6.1218

Cacioppo, J. T., Petty, R. E., Kao, C. F. \& Rodriguez, R. (1986). Central and peripheral routes to persuasion: An individual difference perspective. Journal of Personality and Social Psychology, 51, 1032-1043. doi:10.1037/00223514.51.5.1032

Cárdenas, M. \& Barrientos, J. (2008). Actitudes explícitas e implícitas hacia los hombres homosexuales en una muestra de estudiantes universitarios en Chile. Psykhe, 17(2), 17-25. doi:10.4067/S0718-22282008000200002

Cepeda, G. \& Rosas, C. (2007). Entonación versus justificación adverbial como efecto de cortesía. Revista de Lingüistica Teórica y Aplicada, 45(2), 111-133. doi:10.4067/S0718-48832007000200009

Chaiken, S. (1980). Heuristic versus systematic information processing and the use of source versus message cues in persuasion. Journal of Personality and Social Psychology, 39, 752-756. doi:10.1037//0022-3514.39.5.752

Chaiken, S., Liberman, A. \& Eagly, A. H. (1989). Heuristic and systematic information processing within and beyond the persuasion context. En J. S. Uleman \& J. A. Bargh (Eds.), Unintended thought (pp. 212-252). New York, NY: Guilford Press.

Cialdini, R. B. \& Trost, M. R. (1998). Social influence: Social norms, conformity, and compliance. En D. Gilbert, S. Fiske \& G. Lindzey (Eds.), The handbook of social psychology (4ª ed., Vol. 2, pp. 151-192). New York, NY: McGraw Hill.

Clark, H. H. \& Chase, W. G. (1972). On the process of comparing sentences against pictures. Cognitive Psychology, 3, 472-517. doi:10.1016/0010-0285(72)90019-9 
DeBono, K. G. \& Harnish, R. J. (1988). Source expertise, source attractiveness, and the processing of persuasive information: A functional approach. Journal of Personality and Social Psychology, 55, 541-546. doi:10.1037//00223514.55 .4 .541

Domitrescu, D. (1992). Preguntas con multiconstituyentes interrogativos en español. Hispania, 1, 164-170.

Ellis, A. (1962). Reason and emotion in psychotherapy. New York, NY: Lyle Stuart.

Escandell, M. V. (1984). La interrogación retórica. Dicenda, 3, 9-37.

Gallardo, I., Briñol, P. \& Salazar, C. (2012). El uso de la negación en preguntas retóricas y persuasión. Manuscrito no publicado, Universidad de Talca, Chile/Universidad Autónoma de Madrid, España.

Gibbs, R. W. (2000). Irony in talk among friends. Metaphor and Symbol, 15, 5-27. doi:10.1207/S15327868MS151\&2_2

González, M. J. (2011). La expresión lingüística de la actitud en el género de opinión: El modelo de la valoración. Revista de Lingüistica Teórica y Aplicada, 49(1), 109-141. doi:10.4067/S0718-48832011000100006

Greenwald, A. (1968). Cognitive learning, cognitive response to persuasion, and attitude change. En A. G. Greenwald, T. C. Brock \& T. M. Ostrom (Eds.), Psychological foundations of attitudes (pp. 147-170). New York, NY: Academic Press.

Gutiérrez, J. (1998). Rhetorical questions, relevance and scales. Revista Alicantina de Estudios Ingleses, 11, $139-155$.

Higgins, E. T. (2000). Social cognition: Learning about what matters in the social world. European Journal of Social Psychology, 30, 3-39. doi:10.1002/(SICI)1099-0992(200001/02)30:1<3::AID-EJSP987>3.0.CO;2-I

Horcajo, J., Petty, R. E. \& Briñol, P. (2010). The effects of majority versus minority source status on persuasion: A selfvalidation analysis. Journal of Personality and Social Psychology, 99, 498-512. doi:10.1037/a0018626

Hovland, C. I., Janis, I. L. \& Kelley, H. H. (1953). Communication and persuasion: Psychological studies of opinion change. New Haven, CT: Yale University Press.

Howard, D. J. (1990). Rhetorical question effects on message processing and persuasion: The role of information availability and the elicitation of judgment. Journal of Experimental Social Psychology, 26, $217-239$. doi:10.1016/0022-1031(90)90036-L

Howard, D. J. (1991). The positioning of rhetorical and non-rhetorical questions and the use of self-referencing in print advertising. Journal of Business and Psychology, 5, 397-410. doi:10.1007/BF01017710

Igualada, D. A. (1994). Estrategias comunicativas: la pregunta retórica en español. Revista Española de Lingüistica, 24, 329-344.

Jost, J. T., Kruglanski, A. W. \& Nelson, T. O. (1998). Social metacognition: An expansionist review. Personality and Social Psychology Review, 2, 137-154. doi:10.1207/s15327957pspr0202_6

Just, M. A. \& Carpenter, P. A. (1976). Eye fixations and cognitive processes. Cognitive Psychology, 8, 441-480. doi:10.1016/0010-0285(76)90015-3

Kruglanski, A. W., Raviv, A., Bar-Tal, D., Raviv, A., Sharvit, K., Ellis, S. ... Mannetti, L. (2005). Says who? Epistemic authority effects in social judgment. Advances in Experimental Social Psychology, 37, 346-392. doi:10.1016/S00652601(05)37006-7

Lea, R. B. \& Mulligan, E. J. (2002). The effect of negation on deductive inferences. Journal of Experimental Psychology: Learning, Memory, and Cognition, 28, 303-317. doi:10.1037//0278-7393.28.2.303

Mayo, R., Schul, Y. \& Burnstein, E. (2004). "I am not guilty" versus "I am innocent": Successful negation may depend on the schema used for its encoding. Journal of Experimental Social Psychology, 40, 433-449. doi:10.1016/ j.jesp.2003.07.008

Munch, J. M. \& Swasy, J. L. (1988). Rhetorical questions, summarization frequency, and argument strength effects on recall. Journal of Consumer Research, 15, 69-76. doi:10.1086/209146

Petty, R. E. \& Briñol, P. (2010). Attitude change. En R. F. Baumeister \& E. J. Finkel (Eds.), Advanced social psychology: The state of the science (pp. 217-259). Oxford, Reino Unido: Oxford University Press.

Petty, R. E., Briñol, P. \& Tormala, Z. L. (2002). Thought confidence as a determinant of persuasion: The self-validation hypothesis. Journal of Personality and Social Psychology, 82, 722-741. doi:10.1037//0022-3514.82.5.722

Petty, R. E. \& Cacioppo, J. T. (1986). Communication and persuasion: Central and peripheral routes to attitude change. New York, NY: Springer-Verlag.

Petty, R. E., Cacioppo, J. T. \& Heesacker, M. (1981). Effects of rhetorical questions in persuasion: A cognitive response analysis. Journal of Personality and Social Psychology, 40, 432-440. doi:10.1037//0022-3514.40.3.432

Petty, R. E., Ostrom, T. M. \& Brock, T. C. (1981). Cognitive responses in persuasion: Historical foundations of the cognitive response approach to attitudes and persuasion. En R. E. Petty, T. M. Ostrom \& T. C. Brock (Eds.), Cognitive responses in persuasion (pp. 5-29). Hillsdale, NJ: Lawrence Erlbaum.

Petty, R. E. \& Wegener, D. T. (1998). Attitude change: Multiple roles for persuasion variables. En D. T. Gilbert, S. T. Fiske \& G. Lindsey (Eds.), The handbook of social psychology (4ª ed., Vol. 1, pp. 323-390). New York, NY: McGraw Hill.

Roskos-Ewoldsen, D. R. (2003). What is the role of rhetorical question in persuasion? En J. Bryant, D. R. RoskosEwoldsen \& J. Cantor (Eds.), Communication and emotion: Essays in honor of Dolf Zillmann (pp. 297-322). Hillsdale, NJ: Lawrence Erlbaum.

Salazar, C. (2010). Preguntas retóricas: efectos sobre el cambio de actitudes (Tesis de Magíster no publicada), Facultad de Psicología, Universidad de Talca, Chile.

Schwarz, N., Bless, H., Strack, F., Klumpp, G., Rittenauer-Schatka, H. \& Simons, A. (1991). Ease of retrieval as information: Another look at the availability heuristic. Journal of Personality and Social Psychology, 61, $195-202$. doi:10.1037/0022-3514.61.2.195 
Swasy, J. L. \& Munch, J. M. (1985). Examining the target of receiver elaborations: Rhetorical question effects on source processing and persuasion. Journal of Consumer Research, 11, 877-886. doi:10.1086/209023

Tormala, Z. L., Briñol, P. \& Petty, R. E. (2006). When credibility attacks: The reverse impact of source credibility on persuasion. Journal of Experimental Social Psychology, 42, 684-691. doi:10.1016/j.jesp.2005.10.005

Wegener, D. T. \& Petty, R. E. (1995). Flexible correction processes in social judgment: The role of naive theories in corrections for perceived bias. Journal of Personality and Social Psychology, 68, 36-51. doi:10.1037/00223514.68.1.36

Wegner, D. M. (1989). White bears and other unwanted thoughts: Suppression, obsession, and the psychology of mental control ( $1^{a}$ ed.). New York, NY: Viking Press.

White, P. R. R. (2003). Beyond modality and hedging: A dialogic view of the language of intersubjective stance. Text \& Talk, 23, 259-284. doi:10.1515/text.2003.011

Zillmann, D. (1972). Rhetorical elicitation of agreement in persuasion. Journal of Personality and Social Psychology, 21, 159-165. doi:10.1037/h0032316

Zillmann, D. \& Cantor, J. R. (1974). Rhetorical elicitation of concession in persuasion. The Journal of Social Psychology, 94, 223-236. doi:10.1080/00224545.1974.9923209

Fecha de recepción: Mayo de 2012.

Fecha de aceptación: Octubre de 2012. 